\title{
Orthodontic Bracket Failure Rates in South-Eastern Nigeria
}

\author{
Okeke AC ${ }^{1 *}$, Folaranmi $\mathbf{N}^{2}$, Utomi $\mathrm{IL}^{3}$ \\ ${ }^{1}$ Department of Child Dental Health, University of Nigeria, Enugu, Nigeria \\ ${ }^{2}$ Department of Child Dental Health, University of Nigeria, Enugu, Nigeria \\ ${ }^{3}$ Department of Child Dental Health, University of Lagos, Nigeria
}

*Corresponding Author: Okeke AC, Department of Child Dental Health, University of Nigeria, Enugu, Nigeria. Email: azubuike.okeke@unn.edu.ng

\begin{abstract}
Background: Orthodontic treatment time duration can be prolonged when orthodontic brackets fail. Bracket failures can be influenced by the type of adhesive used for bonding, since different adhesives have different physical and chemical properties.

Objective: The aims of this study were to compare the bracket failure rates of a self-cure adhesive and a light-cure adhesive from the same manufacturer and also to determine the effect of age and sex on bracket failure rate.

Methodology: Thirty patients (20 males and 10 females) with a mean age of 18.87 years were included in the study. Five hundred and twelve orthodontic brackets were bonded by one operator using a prospective singleblinded split mouth design, with either the Light Bond ${ }^{(R)}$ light-cure or the Rely.a.Bond ${ }^{(R)}$ self-cure adhesives. The patients were followed up for 6 months. Data were analysed using frequency, percentage, mean statistics, t-test, analysis of variance (ANOVA) correlation and multiple linear regression analyses. A p-value of 0.05 or less was considered statistically significant.
\end{abstract}

Results: The bracket failure rate for the light-cure adhesive was (9.0\%) while that for the self-cure adhesive was 7.8\%. This difference was however, not statistically significant ( $p$-value=0.624). Age was not a significant predictor of bracket failure rate of either the light-cure adhesive ( $p$-value $=0.794)$ or the self-cure adhesive ( $p$-value=0.994). Similarly, sex was not a significant influence on bracket failure rate of either the light-cure adhesive ( $p$-value=0.941) or the self-cure adhesive ( $p$-value=0.581).

Conclusion: The results of this randomized clinical study demonstrated a higher rate of bracket failure with the use of the light-cure (Light Bond $\left.{ }^{(R)}\right)$ adhesive compared to the self-cure (Rely.a.Bond ${ }^{(R)}$ ). However, this difference was not statistically significant. Age and sex were not significant determinants of bracket failure rate when either the light-cure adhesive or the self-cure adhesive was used.

Keywords: Bracket; Failure; Self-cure; Light-cure; Adhesive

\section{INTRODUCTION}

The use of the acid etch technique in orthodontics, presents the major challenge of orthodontic bracket bond failures in the course of treatment. ${ }^{1}$ An orthodontic bracket bond failure is said to have occurred when the bracket attachment to the enamel surface of a tooth ceases to be attached by the adhesive bond. ${ }^{1}$ These orthodontic bracket failures are relatively frequent, undesirable and has unwanted consequences. Periodontal health may be compromised due to plaque accumulation between the failed bracket and the tooth surface. There is usually an increased demand for clinic and clinicians time since the orthodontist will need to follow a protocol to replace the failed bracket. ${ }^{2,3,4}$ There is also an increase in the total treatment time because the tooth on which the bracket has failed cannot be acted upon by the appliance. ${ }^{2,3,4}$ Finally, enamel fractures may also result as a consequence of orthodontic brackets failure. $^{5}$

Several reasons account for bracket failure among which are operator-related factors like the bonding technique, patient-related factors including age, sex, general level of cooperation and compliance with dietary and oral hygiene instructions. ${ }^{6}$ There are also material-related factors like the type of etchant or adhesive used alongside the bracket properties. ${ }^{6}$ Efforts at 
reducing bracket failure rates have been directed at improvements in bonding technique, bracket bases and in adhesive technology. Though manufacturers of orthodontic adhesives continue to claim to have made new advancements as regards clinical efficiency so as to reduce bracket failures, clinical studies are necessary in assessing adhesive performance because all the factors that can contribute to bond failure would be present. ${ }^{7,8}$

The first and most popular bonding resins introduced were the self-curing bonding systems. ${ }^{9} \quad$ The two-paste-mix self-curing polymeric resin adhesives were invented in the 1970 's for direct bonding of attachments to etched enamel surface. ${ }^{10}$ This was followed in 1975 by the invention of the no-mix self-cure adhesive which eliminated the clumsiness of the mixing steps in the two-mix system. ${ }^{11}$ The first single-paste ultraviolet light-curing adhesive was introduced in $1974,{ }^{11}$ however, its use for orthodontic bonding was first described in 1979. ${ }^{12}$ Light-cure adhesives polymerize due to a reaction between the catalyst in the adhesive and the photon emitted by the light-curing source. The evolution of the light source has been from bulky, corded halogen curing lamps to lightweight, portable, Light-emitting diodes (LED) lights. ${ }^{13}$

\section{Materials ANd Methods}

This study was carried out at the Orthodontic unit of the Department of Child Dental Health, University of Nigeria Teaching Hospital (UNTH), Enugu, Nigeria after ethical approval was obtained from the Ethics and Research Committee of the University of Nigeria Teaching Hospital, Ituku-Ozalla, Enugu with approval number 1RB00002323.

Written consent was obtained from parents while the children also gave assent. It was a prospective six-month, single-blinded split mouth design in which the rates of orthodontic bracket failure was determined after using Light Bond $^{(\mathrm{R})}$ light-cure and Rely.a.Bond ${ }^{(\mathrm{R})}$ self-cure adhesives to bond brackets on a total of five hundred and twelve (512) teeth, with two hundred and fifty six (256) brackets bonded using each adhesive type in 30 consecutive patients whose treatment with upper and lower fixed appliance therapy required or did not require tooth/teeth extractions. The study was not limited to any type of malocclusion, however, patients who had teeth with fractured or restored buccal surface, microdonts or enamel hypoplasia were excluded from the study.
Teeth of consecutive male and female participants presenting at the Orthodontic Unit of the UNTH, which met the inclusion criteria for the study were bonded during treatment. The bonding procedure was performed by the same clinician to avoid possible procedural differences. It was a prospective randomized clinical trial which used a single-blind design, involving a within-subject comparison of the light-cure and the self-cure adhesive systems, with each subject randomly allocated two bonding systems for each side of the mouth. The Battenburg design was used as in a previous study ${ }^{4}$ in which for a participant, when upper right/ lower left quadrants were bonded with one type of adhesive, then the upper left/lower right quadrants were bonded with the other type of adhesive. This design had the advantage that both adhesive types were on each side of a participant's mouth, allowing both to simultaneously experience similar intra-oral conditions on both sides of the mouth. Bonding of brackets to teeth was carried out by one operator from the Central Incisors to the Second Premolars. (Where any tooth had been extracted from any quadrant, the remaining teeth were bonded). Bracket failure rates were prospectively determined for each adhesive type.Teeth were scaled and polished using pumice. They were then rinsed with water and dried in a stream of oil-free compressed air. ${ }^{20}$

\subsection{Bonding with the Self-cure Adhesive}

The teeth were isolated using cotton pledgets and cheek retractor, in readiness for bonding. Brush applicator was used to apply $37 \%$ Phosphoric acid gel to the mid-buccal enamel surface of each tooth (from the Central Incisor to the $1^{\text {st }}$ Molar) and left for 15 seconds. In participants who had teeth extracted as part of the treatment plan, the remaining teeth were prepared for bonding. Following adequate etching, the teeth were rinsed with water and dried with oil free compressed air, until the enamel surface appeared frosty white. Rely.a.Bond ${ }^{(\mathrm{R})}$ adhesive primer was applied onto the etched tooth surface and the mesh surface of the bracket using a brush applicator. The Rely.a.Bond ${ }^{(\mathrm{R})}$ adhesive was syringed onto the bracket base (just enough to cover the bracket base when placed on the tooth) and firmly placed in position on the tooth surface. After thirty seconds, excess adhesive was removed using a sharp probe. ${ }^{20} \mathrm{~A}$ waiting period of between 10 minutes was observed to allow for adequate polymerization of the adhesive. ${ }^{14,15}$ 
0.014 Nickel-Titanium archwire was ligated onto the brackets. Same wire sequence was used after bonding for all the patients.

\subsection{Bonding with the Light-cure Adhesive}

Similar manufacturer's recommended protocol as with the self-cure adhesive up to the point of etching was followed. ${ }^{20} \mathrm{~A}$ thin layer of Light Bond $^{(\mathbb{R})}$ adhesive primer was then smeared onto the etched tooth surface with a different brush applicator. Light Bond $^{(\mathrm{R})}$ light-cure adhesive was syringed onto the bracket base and placed in position on the tooth surface. Excess resin was removed by running a dental probe around the base of the bracket. The resin was polymerized using the Woodpecker LED-b curing light source with wavelength of $480 \mathrm{~nm}$ on the tooth (20 seconds for each bracketadhesive interface: 10 seconds on the mesial and 10 seconds on the distal side). The light source was brought as close to the bracket as possible, as was recommended by the manufacturer. 0.014 Nickel-Titanium archwire was ligated onto the brackets. Same wire sequence was used after bonding for all the patients.

\subsection{Post Set-up Instructions and Reviews}

Verbal and written oral hygiene and care of appliance instructions were given to each participant. They were to by brush their teeth with a fluoride containing toothpaste after every meal. They should eat only soft food during the duration of the treatment because hard, large and sticky pieces of food (nuts, crisps, chunky meat and chewing gum) may damage the appliance. They were also to avoid taking a bite, but to cut large pieces of food into smaller pieces before eating them.

Weekly telephone calls were made to each participant throughout the research duration of six months during which they came for review every six weeks and similar sequence of archwire and treatment approach was adopted for each patient.

\subsection{To Determine the Bracket Failure Rate}

Each participant was given a diary in which to record the date and time of bracket failures. They visually inspected the appliance daily using a mirror and reported any loosening of bracket via telephone as soon they thought it occurred and in such circumstance, were asked to report to the clinic as soon as possible for bracket replacement. The type of adhesive that was used to bond each tooth and sex of participant were also recorded. In case of failures not detected and therefore, not recorded by the patient, the date of the review appointment during which the clinician detected the failure, was taken as the date of failure. No second recording of the same tooth was made even if a second bracket failure results for that tooth. At the completion of six months of observation for each participant, the following were determined;

1. The rate of bracket failure with the Selfcure adhesive

2. The rate of bracket failure with the Lightcure adhesive

\subsection{Statistical Analysis}

Analysis of the data was carried out using descriptive statistics (frequency, percentage, mean and standard deviation). Chi-square was used to compare bracket failures and type of adhesives used. Analysis of variance (ANOVA) was used to compare the bracket failure rate among the various age categories. Multiple linear regression analysis was used to ascertain if age and sex were significant predictors of bracket failure rates. A p-value of 0.05 or less was considered statistically significant. Statistical Package of Social Science (SPSS)/Statistical Product for Service Solution (SPSS) version 21.0 was used to analyse the data generated.

The light-cured adhesives have unlimited working time during bracket placement, less patient discomfort because of accelerated setting time and significant less chair side time, since archwire can be placed immediately without having to wait 8-10 minutes for polymerization to occur. ${ }^{14,15}$ Bracket placement and flash removal are also much easier with the light activated composites. ${ }^{14,15}$ One key advantage of the self-cure adhesive is that it does not require the curing light, which makes its useless bulky versatile for use even in rural areas with no power supply.

Previous studies across the world, ${ }^{2,16,17,18}$ had studied failure rates of light-cure and self-cure adhesives, with varying reports including that both adhesive types have different failure rates ${ }^{19}$ and similar failure rates. ${ }^{17,18}$ Bishara et $\mathrm{al}^{17}$, also reported greater use of the light-cure adhesive than the Self-cure adhesive. However, there is still limited information on comparative assessment of bracket failure rates using of the light-cure and the self-cure adhesives, hence the need for this study. 


\section{RESUltS}

As shown in table 1, the participants were predominantly males $(66.7 \%)$, and the

Table1. Percentage Distribution of participant's sex remaining $33.3 \%$ were females. The participants had a mean age of $18.87 \pm 7.27$ years.

\begin{tabular}{|l|l|l|}
\hline Variables & Frequency(n=30) & Percentage \\
\hline Sex & \multicolumn{2}{|c|}{} \\
\hline Male & 20 & 66.7 \\
\hline Female & 10 & 33.3 \\
\hline
\end{tabular}

Table2. Relationship between Bracket Failure Rate and Adhesive Type

\begin{tabular}{|l|l|l|l|l|l|}
\hline $\begin{array}{l}\text { Type of } \\
\text { adhesive }\end{array}$ & $\begin{array}{l}\text { Number } \\
\text { Bonded }\end{array}$ & $\begin{array}{l}\text { Number of bracket } \\
\text { failures }\end{array}$ & $\begin{array}{l}\text { Bracket Failure } \\
\text { rates }\end{array}$ & $\begin{array}{l}\text { Chi-Square } \\
\text { value }\end{array}$ & p-value \\
\hline Light-cure & 256 & 23 & 9.0 & 0.240 & 0.624 \\
\hline Self-cure & 256 & 20 & 7.8 & & \\
\hline
\end{tabular}

Of the 256 teeth bonded with the light-cure adhesive, $23(9.0 \%)$ failed, while the remaining $233(91.0 \%)$ did not fail. While $20(7.8 \%)$ of the brackets bonded on 256 teeth using the self-cure adhesive failed, the remaining $236(92.2 \%)$ did not fail as shown in table 2. Therefore, a total of 43(8.4\%) brackets (of the 512 bonded) failed. Test of association between bond failure and type of orthodontic adhesive used, showed that there was no significant association $\left(X^{2}=0.240\right.$, $\mathrm{p}$-value $=0.624)$. That is, failure rate had no significant association with the type of orthodontic adhesive used.

Regarding the influence of age with the use of the light-cure, participants aged $23-27$ years

Table3. Comparison of Failure Rates among the Age group using ANOVA

\begin{tabular}{|c|c|c|c|}
\hline Adhesive Type & Age group (Years) & Mean Percentage Failure rate (\%) & $\mathrm{p}$-value \\
\hline \multirow{5}{*}{ Light-cure } & $13-17$ & 10.0 & \multirow{5}{*}{0.708} \\
\hline & $18-22$ & 3.1 & \\
\hline & $23-27$ & 12.5 & \\
\hline & $28-32$ & 6.3 & \\
\hline & $33-37$ & 10.8 & \\
\hline \multirow{5}{*}{ Self-cure } & $13-17$ & 7.5 & \multirow{5}{*}{0.616} \\
\hline & $18-22$ & 12.5 & \\
\hline & $23-27$ & 0.0 & \\
\hline & $28-32$ & 0.0 & \\
\hline & $33-37$ & 11.7 & \\
\hline
\end{tabular}

Teeth bonded with light-cure had more percentage failure rate among the males $(9.1 \%)$ as against their female counterparts who had an average percentage failure rate of $8.8 \%$. A further comparison among the males and females showed no significant difference in the mean percentage failure rates $(p=0.918)$. With the self-cure adhesive, a similar pattern was observed. The male participants had a higher average percentage failure of $8.6 \%$, whereas the females had $6.3 \%$ as their average failure rates. The comparison between genders showed that had the highest mean percentage failure $(12.5 \%)$, while those aged $18-22$ years had the least mean percentage failure rate $(3.1 \%)$. A comparison of the mean percentage failure rate showed no significant difference across the various age categories $(p=0.708)$. With the selfcure adhesive, participants aged 18-22 years had the highest mean percentage failure rate (12.5\%). While both 23-27 years and 28-32 years age ranges had no $(0.0 \%)$ percentage failure. The comparison showed that age ranges had no significant differences on their mean percentage failure $(\mathrm{p}=0.616)$ as shown in table 3.

there is no significant difference in the mean percentage failure rates $(\mathrm{p}=0.572)$. (Table 4)

It is seen in table 5 that the light-cure adhesive, age was not found to be a significant determinant of bracket percentage failure $(\mathrm{P}=0.794)$. Also, sex was not found to be a significant predictor of the bracket percentage failure $(\mathrm{P}=0.941)$. The test of both age and sex together as possible determinant of bracket percentage failure was not also significant $(\mathrm{P}=0.961)$ with an adjusted $\mathrm{R}^{2}$ value of 0.071 . 
Table4. Comparison of Bracket Failure Rates between Males and Females using t-test

\begin{tabular}{|l|l|l|l|}
\hline Variables & Sex & Mean Bracket failure rate (\%) & p- value \\
\hline \multirow{2}{*}{ Light-cure } & Male & 9.1 & 0.918 \\
\cline { 2 - 3 } & Female & 8.8 & \\
\hline \multirow{2}{*}{ Self-cure } & Male & 8.6 & \multirow{2}{*}{0.572} \\
\cline { 2 - 3 } & Female & 6.3 & \\
\hline
\end{tabular}

Table5. Multiple Linear Regression Analysis of the Influence of Age and Sex on Bracket Failure Rate of the Light-cure Adhesive

\begin{tabular}{|c|c|c|c|c|c|c|c|}
\hline & \multicolumn{2}{|c|}{$\begin{array}{l}\text { Unstandardized } \\
\text { Coefficients }\end{array}$} & \multirow{2}{*}{\begin{tabular}{|l|} 
Standardized \\
Coefficients
\end{tabular}} & \multirow[t]{2}{*}{ T- Values } & \multirow[t]{2}{*}{ p-value } & \multicolumn{2}{|c|}{$95 \%$ Confidence Interval } \\
\hline & B & Std. Error & & & & Lower & Upper \\
\hline Constant & 10.032 & 5.726 & & 1.752 & 0.091 & -0.305 & 23.215 \\
\hline Age & -0.064 & 0.245 & -0.051 & -0.263 & 0.794 & -0.510 & 0.246 \\
\hline Sex & 0.275 & 3.709 & 0.014 & 0.074 & 0.941 & -7.373 & 7.430 \\
\hline
\end{tabular}

Adjusted $R^{2}=0.071 ; p=0.961$

The relationship between the age and sex and failure rate among patients who received the self-cure adhesive. Both age and sex did not significantly predict $(\mathrm{p}=0.855)$ the bracket percentage failure with adjusted $\mathrm{R}^{2}=0.062$. (Table 6)

Table6. Multiple Linear Regression Analysis of the Influence of Age and Sex on Bracket Failure Rate of selfcure adhesive

\begin{tabular}{|l|l|l|l|l|l|l|l|}
\hline & \multicolumn{2}{|l}{$\begin{array}{l}\text { Unstandardized } \\
\text { Coefficients }\end{array}$} & $\begin{array}{l}\text { Standardized } \\
\text { Coefficients }\end{array}$ & \multirow{2}{*}{ T value } & p-value & \multicolumn{2}{l|}{ 95\% Confidence Interval } \\
\cline { 2 - 3 } \cline { 7 - 9 } & B & Std. Error & Beta & & & Lower & Upper \\
\hline Constant & 6.206 & 6.566 & & 0.945 & 0.353 & -7.101 & 20.500 \\
\hline Age & 0.002 & 0.281 & 0.002 & 0.008 & 0.994 & -0.589 & 0.532 \\
\hline Sex & 2.378 & 4.253 & 0.108 & 0.559 & 0.581 & -6.932 & 10.128 \\
\hline
\end{tabular}

Adjusted $R^{2}=0.062 ; p=0.855$

\section{DISCUSSION}

In the present study, the bracket failure rate for the light-cure adhesive was more than that for the self-cure adhesive, but the difference was not significant. Age and Sex were also not found to be significant predictors of bracket failure rate. The bracket failure rate for the light-cure adhesive in this study was $9.0 \%$ which is similar to that reported by Galindo et $\mathrm{al}^{21}$ in which the failure rate was $11.3 \%$ after a study duration of eleven months. Le et $\mathrm{al}^{22}$ had also reported a value of $11.3 \%$. Conversely, O'Brien et $\mathrm{al}^{23}$ and Millet et $\mathrm{al}^{24}$ reported a relatively much lower bracket failure rate. Following a study duration that lasted through the entire treatment time, O'Brien et $\mathrm{al}^{23}$ reported a failure rate of $3.9 \%$ while Millet et $\mathrm{al}^{24}$ reported a failure rate of $6 \%$ over a 48 -month study period. The lower value reported in the previous studies ${ }^{23,24}$ maybe as a result of dietary differences which have also been reported to affect bond failure rate. ${ }^{1}$ The harder the diet, the greater the tendency for failure to occur. ${ }^{1}$
With the self-cure adhesive used in the present study, the bracket failure rate was $7.8 \%$, which is similar to the finding by $\mathrm{O}^{\prime} \mathrm{Brien}$ et $\mathrm{al}^{23}$ in which the bracket failure rate for the self-cure adhesive was $7.5 \%$. However, in a Nigeria based study ${ }^{25}$ which used similar adhesive to the present study, Moninuola et $\mathrm{al}^{25}$ reported a higher failure rate of $24.1 \%$. This higher value of the self-cure adhesive may be attributed to the longer study duration of 24 months as against 6 months in the present study. In the present study, when the bracket failure rates of the light and the self-cure adhesive systems in the clinic were compared within the study duration of 6 months, greater bracket failure rate was recorded for the light-cure than for the selfcure adhesives. This higher failure rate for the light-cure adhesive has been reported in a previous study by Trimpeneers et $\mathrm{al}^{18}$ who reported a significantly higher failure rate for light-cure (24.3\%), being twice that for self-cure $(12.4 \%)$. However, the higher bracket failure rate recorded for the light-cure adhesive in the present study was not statistically significant. 
Incomplete polymerization has been suggested as a possible reason for this higher bracket failure rate with the light-cure adhesives. ${ }^{26,27} \mathrm{On}$ the other hand, other studies reported higher failure rates for self-cure adhesives.,21,23. Galindo et $\mathrm{al}^{21}$ reported a $12 \%$ bracket failure rate for the self-cure and $11.3 \%$ for the lightcure. Likewise, in a separate study by O'Brien et $\mathrm{al}^{23}$, a failure rate of $7.5 \%$ and $5.5 \%$ was reported for self-cure and light-cure, respectively. However, Sonis et $\mathrm{al}^{14}$ and Artun et $\mathrm{al}^{28}$, reported no significant difference in bracket failure rate between the light and the self-cure adhesives. This is in agreement with the findings in the present study which showed that despite the higher failure rate of the lightcure adhesive over the self-cure, the difference was not significant.

Following the reported presence of confounding factors, the effects of age and sex on the failure rates of the light-cure and self-cure adhesives were also investigated in the clinic study. ${ }^{25,29,30}$ Investigation of the relationship between age and bracket failure rate of either the light-cure adhesive or the self-cure adhesive, showed no significant relationship. One study ${ }^{24}$ had reported similar finding, though the same group of researchers had in an earlier study ${ }^{31}$ reported lower brackets failure rates in adults than in younger patients. Other studies have also reported a higher failure rate in the younger age group than in the adults. ${ }^{25,29,30,32}$ This has been attributed to increased self-consciousness and self-motivation in adults while undergoing orthodontic treatment when compared to adolescents. ${ }^{29,32}$ However, the finding of a more recent study ${ }^{31}$ agrees with the finding in the present study, that age does not necessarily dictate bracket failure rate.

In the present study, sex did not significantly affect the bracket failure rates, however males had greater failure rate than the females. This finding agrees with that of previous studies carried out in Nigeria ${ }^{25,30,34}$ which reported that males have a higher failure rate of brackets. A Nigerian based study by Moninuola et $\mathrm{al}^{25}$ reported the bracket failure rate in males as $26.2 \%$ and in females $23.4 \%$. Aikins and Ututu ${ }^{30}$ in a later study carried out at a tertiary health facility, reported a bracket failure rate of $81.2 \%$ for males and $69.2 \%$ for females. A similar finding has also been reported in a previous study in Europe, ${ }^{35}$ with males having 2.4 times greater chance of bracket failure than females. The reasons for the greater bracket failure rate in males may be as a result of a greater care for oral hygiene exhibited by the females, including the fact that females tend to apply lighter masticatory forces than males ${ }^{30,36,37}$. Other possible explanations are that males engage more in contact sports which can predispose to traumatic bracket failure. ${ }^{36}$ Males are also said to practice bad eating habits than females since they eat more of junk food, which may contain hard food particles that may break off brackets. ${ }^{36}$

Notwithstanding the above reported findings, contradictory results have been reported in which females were reported as having greater bracket failures. ${ }^{29,32,36}$ Though higher failure rate was noted for males in this study, sex was not a significant predictor of bracket failure rates of light-cure adhesives nor is it for the self-cure adhesive. This is similar to the report from several studies $16,33,38,39,40,41,42$ which showed that sex was not a significant predictor of bracket failure rates. Umeh et al in their study ${ }^{41}$ carried out in Lagos, Nigeria reported no significant effect of gender, time, or side of the arch on the prevalence of first molar buccal tube failure.

\section{CONClusion}

The bracket failure rate of the light-cure adhesive was higher than that of the self-cure adhesive. Age had no significant influence on the bracket failure rates of orthodontic brackets bonded with either the light-cure or the self-cure adhesive. Sex had no significant influence on the bracket failure rates of orthodontic brackets bonded with either the light-cure or the self-cure adhesive.

\section{LIMITATION}

The study only assessed failure rate within the 6 months' study period. Changes beyond this period which may be different from the reported findings are possible.

\section{REFERENCES}

[1] Reis A, Santos JE, Loguercio AD, Bauer JRO. Eighteen-month bracket survival rate: conventional versus self-etch adhesive. Eur J Orthod. 2008; 30:94-99.

[2] Natheer AR. Orthodontic bracket failure rate; a comparative clinical study between light-cured and chemically cured (no mix) bonding systems. J Bagh Coll Dentistry 2012; 24:137139.

[3] Cook A. Curing lights-are you contributing to your bond failure rate? http://www.andreacookconsulting.com/newslett er archives/2010 07/ 
[4] Aljubouri YD, Millett DT, Gilmour WH. Six and 12 months' evaluation of a self-etching primer versus two-stage etch and prime for orthodontic bonding: a randomized clinical trial. Eur J Orthod. 2004: 26:565-571.

[5] Vasudevan A, Antony V, Francis P, Parayaruthottam P, Shaloob M, TP MH. Influence of Patient Attitude on Bracket Failure Rate: A Prospective Study. J Ind Orthod Soc. 2021:1;1-7

[6] Chen CS, Hsu ML, Chang KD, Kuang SH, Chen PT, Gung YW. "Failure analysis: enamel fracture after debonding orthodontic brackets," Angle Orthod. 2008; 6:1071-1077.

[7] Markovic E, Glisic B, Scepan I, Markovic D, Jokanovic V. Bond strength of orthodontic adhesives. Metal J Metall 2011; 14:78-88.

[8] Manuel T, Raquel O, Estrella O, Alejandro R, Blanca H, Franklin G. Bond Strength of Orthodontic Brackets Using Different Light and Self-Curing Cements. Angle Orthod. 2003;73: 56-63.

[9] Turkkahraman HT, Adanir N, Gungor AY, Alkis H. In vitro evaluation of shear bond strengths of colour change adhesives Eur J Orthod. 2010; 32: 571-574.

[10] Gange P. The evolution of bonding in orthodontics. Am J Orthod Dentofacial Orthop. 2015;147: 56-63.

[11] Tavas MA, Watts DC. Bonding of orthodontic brackets by trans- illumination of a light activated composite: an in vitro study. $\mathrm{Br} \mathrm{J}$ Orthod. 1979; 6:207-208.

[12] Krug AY, Canley RS. Shear bond strengths using an indirect technique with different light sources. J. Clin. Orthod. 2005; 39:485-487.

[13] Newman GV, Synder WH, Wilson CE Jr. Acrylic adhesives for bonding attachments to tooth surfaces. Angle Orthod 1968; 38: 12-18.

[14] Sonis AL. Comparison of a light-cured adhesive with an autopolymerization bonding system. J Clin Orthod. 1988; 22: 730-732.

[15] Hamula DW. Technique clinic: Direct bonding with light-cured adhesives. J Clin Orthod 1991; 25: 437-438.

[16] Bherwani A, Fida M. Bond failure with a NoMix Adhesive System. Angle Orthod. 2008; 78:545-548.

[17] Bishara SE, Laffoon JF, VonWald L, Warren J. The effect of repeated bonding on the shear bond strength of different orthodontic adhesives. Am J Orthod Dentofacial Orthop. 2002; 121: 521-525.

[18] Trimpeneers LM, Dermaut LR. A clinical trial comparing the failure rates of two orthodontic bonding systems. Am J Orthod Dentofacial Orthop. 1996; 110:547-550.
[19] Zero DT. How the introduction of the acid-etch technique revolutionized dental practice.J Am Dent Assoc. 2013; 9:990-994.

[20] Lolayekar NV, Bhat VS, Baht SS. Disinfection methods of extracted human teeth. J Oral Health Comm Dent. 2007; 1: 27-29

[21] Galindo HRA, Sadwosky PL, Vlachos CH, Jacobson A, Wallance D. An in vitro comparison between a visible light- cured bonding system and a chemically cured bonding system. Am J Orthod Dentofacial Orthop. 1998; 113: 271-275.

[22] Le PT, Weinstein M, Borislow AJ, Braitman LE. Bond failure and decalcification: A comparison of a cyanoacrylate and a composite resin bonding system in vivo. Am J Orthod Dentofacial Orthop. 2003; 123:624-627

[23] O'Brien KD, Read MJF, Sandison RJ, Roberts CT. A visible light activated direct bonding material: an in-vivo comparative study. Am J Orthod Dentofacial Orthop. 1989; 95: 348-351.

[24] Millett DT, Hallgren A, Cattanach D, McFadzean R, Pattison I, Robertson M, Love J. A 5- year clinical review of bond failure with a light-cured resin adhesive. Angle Orthod. 1998; 68:351-356.

[25] Moninuola AE, daCosta OO, Isiekwe MC. A review of orthodontic bond failure using a chemical cure adhesive. Odontostomatol Trop 2010; 33:35-40.

[26] Rachala MR, Yelampalli MR. Comparison of shear bond strength of orthodontic brackets bonded with light emitting diode (led). Int $\mathrm{J}$ Orthod Milwaukee. 2010; 21:31-35

[27] Shon WJ, Kim TW, Chung SH, Jung MH. The effects of primer precuring on the shear bond strength between gold alloy surfaces and metal brackets. Eur J Orthod. 2012; 34:72-76.

[28] Artun J. A post-treatment evaluation of multibonded ceramic brackets in orthodontics. Eur J Orthod. 1997; 19:219-228.

[29] Rasool G, Raza HA, Afzal F, Ijaz W, Shah SS. Frequency of bracket breakage and bond failure in patients undergoing fixed orthodontic treatment at Khyber college of Dentistry, Peshawar. Pak Oral Dent J 2013; 33:299-302.

[30] Aikins EA, Ututu C. An audit of bonding failure among orthodontic patients in a tertiary hospital in South-South Nigeria. Int J Orthod Rehabil. 2017; 8:91-95.

[31] Millett DT, Gordon PH. A 5-year clinical review of bond failure with a no-mix adhesive (Right on). Eur J Orthod. 1994; 16:203-211

[32] Sukhia HR, Sukhia RH, Mahar A. Bracket debonding and breakage prevalence in orthodontic patients. Pak Oral Dent J. 2011; 31:73-77. 
[33] Roelofs T, Merkens N, Roelofs J, Bronkhorst $\mathrm{E}$, Breuning H. A retrospective survey of the causes of bracket- and tube-bonding failures. Angle Orthod. 2017; 87:111-117.

[34] Ogbonna CM. A comparative evaluation of two orthodontic bonding systems [Unpublished dissertation]. West African College of Surgeons; 2018.

[35] Murfitt PG, Quick AN, Swain MV, Herbison GP. A randomized clinical trial to investigate bond failure rates using a self-etching primer. Eur J Orthod. 2006; 28: 444-449.

[36] Al Duliamy MJ. The effect of oral hygiene status on the bond failure rate of the orthodontic bracket: An in vivo clinical study. J. Dent. Res. 2018; 5: 1-12

[37] Adolfsson U, Larsson E, Ogaard B. Bond failure of a no-mix adhesive during orthodontic treatment. Am J Orthod Dentofacial Orthop. 2002;122: 277-281.
[38] Elekdag-Turk S, Isci D, Turk T, Cakmak F. Six month bracket failure rate evaluation of selfetching primer. Eur J Orthod. 2008; 30; 211 216.

[39] Pandis N, Polychronopoulos A, Eliades T. Failure rate of sel-ligating and edgewise brackets bonded with a conventional acid etching and self etching primer. Angle Orthod. 2006; 76:119-122.

[40] Elekdag-Turk S, Cakmak F, Isci D, Turk T. 12month self-ligating bracket failure rate with a self-etching primer. Angle Orthod. 2008; 78: 1095-1100.

[41] Umeh OD, Eniola AS, Ndukwe AN. A 3-year audit of the failure rate of first molar buccal tubes among orthodontic patients in a Nigerian population. Niger J Med 2021;30:205-209.

[42] Linklater R, Gordon P. Bond failure patterns in vivo. Am J Orthod Dentofacial Orthop. 2003; 123:534-539

Citation: Okeke AC, Folaranmi N, Utomi IL. "Orthodontic Bracket Failure Rates in South-Eastern Nigeria”. ARC Journal of Dental Science. 2021; 6(1):29-36. DOI: https://doi.org/10.20431/2456-0030.0601005.

Copyright: (C) 2021 Authors. This is an open-access article distributed under the terms of the Creative Commons Attribution License, which permits unrestricted use, distribution, and reproduction in any medium, provided the original author and source are credited. 